\title{
ISOMETRIC ACTIONS ON THE PROJECTIVE PLANES AND EMBEDDED GENERATORS OF HOMOTOPY GROUPS
}

\author{
THOMAS PÜTTMANN AND A. RIGAS
}

\begin{abstract}
We determine all orbits of isometric actions on $\mathbb{C P}^{2}, \mathbb{H} \mathbb{P}^{2}$, and $\mathbb{O} \mathbb{P}^{2}$ that are covered by spheres and represent nontrivial classes in the corresponding homotopy groups. As new examples we find orbits that represent generators of $\pi_{5}\left(\mathbb{H} \mathbb{P}^{2}\right) \approx \mathbb{Z}_{2}$ and $\pi_{11}\left(\mathbb{O P}^{2}\right) \approx \mathbb{Z}_{24}$. These orbits are the quaternionic and octonionic analogues of the quadric $z_{1}^{2}+z_{2}^{2}+z_{3}^{2}=0$ in $\mathbb{C P}^{2}$.
\end{abstract}

\section{INTRODUCTION}

It is a classical problem to represent elements of the homotopy groups $\pi_{k}(M)$ of a compact manifold $M$ by geometrically nice maps. In this paper we consider the projective planes $\mathbb{C P}^{2}, \mathbb{H} \mathbb{P}^{2}$, and $\mathbb{O P}^{2}$. It is well-known that projective lines represent generators of the first nontrivial groups $\pi_{2}\left(\mathbb{C P}^{2}\right), \pi_{4}\left(\mathbb{H} \mathbb{P}^{2}\right), \pi_{8}\left(\mathbb{O P}^{2}\right)$. Any projective line is the cut locus of a point $p$ and this cut locus is an isolated singular orbit of the action of the isotropy group at $p$. Therefore, it is natural to ask whether there are other orbits $\mathcal{O}$ of isometric actions such that there exists a covering map $\mathbb{S}^{k} \rightarrow \mathcal{O}$ which represents a nontrivial element in $\pi_{k}\left(\mathbb{C P}^{2}\right), \pi_{k}\left(\mathbb{H} \mathbb{P}^{2}\right), \pi_{k}\left(\mathbb{O} \mathbb{P}^{2}\right)$.

Theorem. Any such orbit $\mathcal{O}$ is up to congruence one of the following:

- A projective line in $\mathbb{C P}^{2}, \mathbb{H}^{2}$, or $\mathbb{O P}^{2}$.

- The quadric $z_{1}^{2}+z_{2}^{2}+z_{3}^{2}=0$ in $\mathbb{C P}^{2}$ or the analogous orbits in $\mathbb{H} \mathbb{P}^{2}$ and $\mathbb{O P}^{2}$. These orbits $S_{\mathbb{C}}, S_{\mathbb{H}}$, and $S_{\mathbb{O}}$ are diffeomorphic to $\mathbb{S}^{2}, \mathbb{S}^{5}$, and $\mathbb{S}^{11}$. They represent twice a generator of $\pi_{2}\left(\mathbb{C P}^{2}\right) \approx \mathbb{Z}$, the generator of $\pi_{5}\left(\mathbb{H} \mathbb{P}^{2}\right) \approx \mathbb{Z}_{2}$, and a generator of $\pi_{11}\left(\mathbb{O P}^{2}\right) \approx \mathbb{Z}_{24}$.

The important part of the theorem is that the orbits $S_{\mathbb{H}}$ and $S_{\mathbb{O}}$ generate $\pi_{5}\left(\mathbb{H} \mathbb{P}^{2}\right)$ and $\pi_{11}\left(\mathbb{O P}^{2}\right)$. This result is interesting in the following two respects: First, the orbits $S_{\mathbb{H}}$ and $S_{\mathbb{O}}$ are isolated singular orbits and hence minimal submanifolds (in contrast to the quadric, however, they are not stable minimal, see Ohnita's classification [11]). Second, the well-known fact that the quadric represents twice a generator of $\pi_{2}\left(\mathbb{C P}^{2}\right) \approx H_{2}\left(\mathbb{C P}^{2}\right)$ is usually considered to be a homological result rather than a homotopical one because of its homological generalizations in algebraic geometry. Our approach, on the other hand, shows how this fact generalizes to $\mathbb{H} \mathbb{P}^{2}$ and $\mathbb{O P}^{2}$ in a purely homotopical way (note that $H_{5}\left(\mathbb{H}^{2}\right)$ and $H_{11}\left(\mathbb{O P}^{2}\right)$ vanish): We deform parametrizations of the orbits $S_{\mathbb{C}}, S_{\mathbb{H}}$, and $S_{\mathbb{O}}$ by geodesic

2000 Mathematics Subject Classification. Primary 57T20, Secondary 53C35, 53C42, 57S15. Key words and phrases. projective planes, homotopy groups, isometric actions, octonions. The joint work of the authors was supported by the CNPq/GMD-agreement. 
retractions to maps with values in the projective lines $\mathbb{C P}^{1}, \mathbb{H P}^{1}, \mathbb{O P}^{1}$ and obtain suspensions of the Hopf fibrations $\mathbb{S}^{1} \rightarrow \mathbb{R P}^{1}, \mathbb{S}^{3} \rightarrow \mathbb{C P}^{1}$, and $\mathbb{S}^{7} \rightarrow \mathbb{H} \mathbb{P}^{1}$. These suspensions are known to represent twice a generator of $\pi_{2}\left(\mathbb{C P}^{1}\right)$, the generator of $\pi_{5}\left(\mathbb{H} \mathbb{P}^{1}\right)$, and a generator of $\pi_{11}\left(\mathbb{O P}^{1}\right)$.

It is a striking fact that the geodesic deformations actually yield maps whose homotopy classes can be determined. This obviously requires a carefully arranged calculation, which finally appears to be rather short. More sophisticated arguments that worked well in the case of $\mathbb{H} \mathbb{P}^{2}$ failed to resolve whether the orbit $S_{\mathbb{O}}$ represents a generator of $\pi_{11}\left(\mathbb{O P}^{2}\right)$ or three times a generator. The explicit homotopy that we supply yields the most precise information possible, since it determines which of the eight generators of $\pi_{11}\left(\mathbb{O P}^{2}\right)$ the orbit $S_{\mathbb{O}}$ represents.

\section{Deformation of parametrizations of the orbits $S_{\mathbb{C}}, S_{\mathbb{H}}$, And $S_{\mathbb{O}}$}

The orbits $S_{\mathbb{C}}, S_{\mathbb{H}}$, and $S_{\mathbb{O}}$ can be described in the following unified way: They are the sets of points with maximal distance to $\mathbb{R P}^{2} \subset \mathbb{C P}^{2}, \mathbb{C P}^{2} \subset \mathbb{H P}^{2}$, and $\mathbb{H} \mathbb{P}^{2} \subset \mathbb{O P}^{2}$, respectively. In this section we parametrize these orbits and deform the parametrizations to maps with values in projective lines. In order to do this we use the Veronese-Jordan models of the projective planes.

We first describe these models. Let $\mathbb{H}=\mathbb{C} \oplus \mathbb{C} j$ denote the quaternions and $\mathbb{O}=\mathbb{H} \oplus \mathbb{H} e$ the octonions with multiplication

$$
\left(u_{1}+v_{1} e\right) \cdot\left(u_{2}+v_{2} e\right)=u_{1} u_{2}-\bar{v}_{2} v_{1}+\left(v_{2} u_{1}+v_{1} \bar{u}_{2}\right) e
$$

and conjugation $u+v e \mapsto \bar{u}-v e$. We equip the hermitian $3 \times 3$-matrices $\operatorname{Herm}_{\mathbb{C}}(3)$, $\operatorname{Herm}_{\mathbb{H}}(3)$, and $\operatorname{Herm}_{\mathbb{O}}(3)$ with the Jordan product $X \circ Y=\frac{1}{2}(X Y+Y X)$. The submanifolds of idempotent elements with trace 1 in these Jordan algebras are diffeomorphic to the projective planes $\mathbb{C P}^{2}, \mathbb{H P}^{2}, \mathbb{O P}^{2}$. In the first two cases the diffeomorphisms are given by the classical Veronese embeddings of $\mathbb{C P}^{2}$ and $\mathbb{H P}^{2}$, i.e., by the maps

$$
\begin{aligned}
\mathbb{C}^{3} \supset \mathbb{S}^{5} \longrightarrow \operatorname{Herm}_{\mathbb{C}}(3), & z \mapsto z \bar{z}^{t}, \\
\mathbb{H}^{3} \supset \mathbb{S}^{11} \longrightarrow \operatorname{Herm}_{\mathbb{H}}(3), & u \mapsto u \bar{u}^{t} .
\end{aligned}
$$

The octonionic case is due to Jordan [10] (see also [2], [5], [6], or [15]).

For technical reasons we apply the affine transformation $X \mapsto 2 X-\mathbb{1}$ to the spaces $\operatorname{Herm}_{\mathbb{C}}(3), \operatorname{Herm}_{\mathbb{H}}(3)$, and $\operatorname{Herm}_{\mathbb{O}}(3)$. This transformation maps the submanifolds of idempotent elements with trace 1 bijectively to the submanifolds of matrices with trace -1 whose square is the identity matrix $\mathbb{1}$. For convenience we still denote the latter submanifolds by $\mathbb{C P}^{2}, \mathbb{H}^{2}$, and $\mathbb{O P}^{2}$. 
The following embeddings turn out to be parametrizations of the orbits $S_{\mathbb{C}}, S_{\mathbb{H}}$, and $S_{\mathbb{O}}$ :

$$
\begin{aligned}
& \iota_{\mathbb{C}}: \mathbb{S}^{2} \hookrightarrow \mathbb{C P}^{2}, \quad x=\left(\begin{array}{l}
x_{1} \\
x_{2} \\
x_{3}
\end{array}\right) \mapsto-x x^{t}+\left(\begin{array}{rrr}
0 & -x_{3} & x_{2} \\
x_{3} & 0 & -x_{1} \\
-x_{2} & x_{1} & 0
\end{array}\right) i \\
& \iota_{\mathbb{H}}: \mathbb{S}^{5} \hookrightarrow \mathbb{H} \mathbb{P}^{2}, \quad z=\left(\begin{array}{c}
z_{1} \\
z_{2} \\
z_{3}
\end{array}\right) \mapsto-z \bar{z}^{t}+\left(\begin{array}{rrr}
0 & -\bar{z}_{3} & \bar{z}_{2} \\
\bar{z}_{3} & 0 & -\bar{z}_{1} \\
-\bar{z}_{2} & \bar{z}_{1} & 0
\end{array}\right) j \\
& \iota_{\mathbb{O}}: \mathbb{S}^{11} \hookrightarrow \mathbb{O P}^{2}, \quad u=\left(\begin{array}{l}
u_{1} \\
u_{2} \\
u_{3}
\end{array}\right) \mapsto-u \bar{u}^{t}+\left(\begin{array}{ccc}
0 & -\bar{u}_{3} & \bar{u}_{2} \\
\bar{u}_{3} & 0 & -\bar{u}_{1} \\
-\bar{u}_{2} & \bar{u}_{1} & 0
\end{array}\right) e
\end{aligned}
$$

It is straightforward to check that the embedding $\mathbb{S}^{2} \hookrightarrow \mathbb{C P}^{2}$ yields a parametrization of the quadric that is given by the equation $z_{1}^{2}+z_{2}^{2}+z_{3}^{2}=0$ in the standard description of $\mathbb{C P}^{2}$. Note that this equation is not well-defined on $\mathbb{H P}^{2}$ and $\mathbb{O P}^{2}$.

Theorem 1. The embeddings above represent twice a generator of $\pi_{2}\left(\mathbb{C P}^{2}\right) \approx \mathbb{Z}$, the generator of $\pi_{5}\left(\mathbb{H} \mathbb{P}^{2}\right) \approx \mathbb{Z}_{2}$, and a generator of $\pi_{11}\left(\mathbb{O} \mathbb{P}^{2}\right) \approx \mathbb{Z}_{24}$, respectively.

Proof. The embedding $\iota_{\mathbb{O}}: \mathbb{S}^{11} \hookrightarrow \mathbb{O P}^{2}$ does not meet the point

$$
p_{0}=\left(\begin{array}{rrr}
1 & 0 & 0 \\
0 & -1 & 0 \\
0 & 0 & -1
\end{array}\right)
$$

Therefore, we can compose it with the geodesic retraction from $\mathbb{O P}^{2} \backslash\left\{p_{0}\right\}$ to the cut locus of $p_{0}$. This geodesic retraction is given by the formula

$$
\left(\begin{array}{ccc}
\xi_{1} & \bar{w}_{3} & w_{2} \\
w_{3} & \xi_{2} & \bar{w}_{1} \\
\bar{w}_{2} & w_{1} & \xi_{3}
\end{array}\right) \mapsto \frac{1}{1-\xi_{1}}\left(\begin{array}{cccc}
\xi_{1}-1 & 0 & 0 \\
0 & \xi_{2}-\xi_{3} & 2 \bar{w}_{1} \\
0 & 2 w_{1} & \xi_{3}-\xi_{2}
\end{array}\right)
$$

The cut locus of $p_{0}$ can be identified with the unit sphere $\mathbb{S}^{8}$ in $\mathbb{R} \times \mathbb{H}^{2}$ by means of the embedding

$$
\mathbb{S}^{8} \hookrightarrow \mathbb{O P}^{2}, \quad\left(\begin{array}{c}
\zeta \\
v_{1} \\
v_{2}
\end{array}\right) \mapsto\left(\begin{array}{ccc}
-1 & 0 & 0 \\
0 & \zeta & v_{1}+v_{2} e \\
0 & \bar{v}_{1}-v_{2} e & -\zeta
\end{array}\right)
$$

All in all, the composition of the embedding $\iota_{\mathbb{O}}$ with the retraction yields the map

$$
\mathbb{S}^{11} \rightarrow \mathbb{S}^{8}, \quad\left(\begin{array}{l}
u_{1} \\
u_{2} \\
u_{3}
\end{array}\right) \mapsto-\frac{1}{1+\left|u_{1}\right|^{2}}\left(\begin{array}{c}
\left|u_{2}\right|^{2}-\left|u_{3}\right|^{2} \\
2 u_{2} \bar{u}_{3} \\
2 \bar{u}_{1}
\end{array}\right)
$$

In order to figure the meaning of this map note that the Hopf fibration $\mathbb{S}^{7} \rightarrow \mathbb{S}^{4}$ can be given by the formula

$$
\left(\begin{array}{c}
u_{2} \\
u_{3}
\end{array}\right) \mapsto\left(\begin{array}{c}
\left|u_{2}\right|^{2}-\left|u_{3}\right|^{2} \\
2 u_{2} \bar{u}_{3}
\end{array}\right)
$$

One can now check that the map given in (5) is (up to sign) the fourth suspension of the map given in (6). It is known [8] that the fourth suspension of the Hopf fibration $\mathbb{S}^{7} \rightarrow \mathbb{S}^{4}$ generates $\pi_{11}\left(\mathbb{S}^{8}\right) \approx \pi_{11}\left(\mathbb{O} \mathbb{P}^{2}\right)$.

We now show that the embeddings above actually are parametrizations of the orbits $S_{\mathbb{C}}, S_{\mathbb{H}}$, and $S_{\mathbb{O}}$. In order to do this we have to describe the actions on $\mathbb{C P}^{2}$, $\mathbb{H} \mathbb{P}^{2}$, and $\mathbb{O P}^{2}$ that leave the totally geodesic subspaces $\mathbb{R P}^{2} \subset \mathbb{C P}^{2}, \mathbb{C P}^{2} \subset \mathbb{H}^{2}$, and $\mathbb{H P}^{2} \subset \mathbb{O P}^{2}$ invariant. These are actions of the groups $\mathrm{SO}(3) \times \mathbb{Z}_{2}, \mathrm{U}(3)$, and $\operatorname{Sp}(3) \times_{\mathbb{Z}_{2}} \operatorname{Sp}(1)$, respectively. For convenience of notation we restrict to the latter action (the other two cases can be treated completely analogously or alternatively 
be carried out in the standard models of $\mathbb{C} \mathbb{P}^{2}$ and $\mathbb{H} \mathbb{P}^{2}$ ). The explicit description of this action was given by Yokota [9]. It is based on the following identification:

$$
\begin{aligned}
\operatorname{Herm}_{\mathbb{H}}(3) \oplus \mathbb{H}^{3} & \rightarrow \operatorname{Herm}_{\mathbb{O}}(3), \\
Q \oplus\left(\begin{array}{l}
u_{1} \\
u_{2} \\
u_{3}
\end{array}\right) & \mapsto Q+\left(\begin{array}{rrr}
0 & -\bar{u}_{3} & \bar{u}_{2} \\
\bar{u}_{3} & 0 & -\bar{u}_{1} \\
-\bar{u}_{2} & \bar{u}_{1} & 0
\end{array}\right) e .
\end{aligned}
$$

Straightforward calculations show that the Jordan product is given by

$$
(Q \oplus u) \circ(Q \oplus u)=\left(Q^{2}-u \bar{u}^{t}+|u|^{2} \mathbb{1}\right) \oplus((\operatorname{tr} Q) u-Q u)
$$

and that $\mathbb{O P}^{2}$ is identified with the set

$$
\left\{\left.Q \oplus u\left|Q^{2}-u \bar{u}^{t}+\right| u\right|^{2} \mathbb{1}=\mathbb{1}, Q u=-u, \operatorname{tr} Q=-1\right\} .
$$

Now $\operatorname{Sp}(3) \times \operatorname{Sp}(1)$ acts by automorphisms on the Jordan algebra $\operatorname{Herm}_{\mathbb{H}}(3) \oplus \mathbb{H}^{3}$ and hence by isometries on $\mathbb{O P}^{2}$ in the following way:

$$
(A, q) \cdot(Q \oplus u)=A Q \bar{A}^{t} \oplus A u \bar{q} .
$$

The kernel of this action is isomorphic to $\mathbb{Z}_{2}$ and the orbit space is a closed interval. In order to give a more detailed description of the orbit space we use the geodesic

$$
\gamma(t)=\left(\begin{array}{ccc}
\cos 2 t & 0 & 0 \\
0 & -\cos 2 t & 0 \\
0 & 0 & -1
\end{array}\right) \oplus\left(\begin{array}{c}
0 \\
0 \\
\sin 2 t
\end{array}\right)
$$

The orbit through $\gamma(0)=p_{0}$ is the totally geodesic subspace $\mathbb{H}^{2} \subset \mathbb{O P}^{2}$. It is easy to see that $\gamma$ intersects this orbit and hence all orbits perpendicularly (by Clairault's theorem the velocity vectors of a geodesic have a constant inner product with a Killing field). The isotropy groups of $\gamma(t)$ for $t \in] 0, \frac{\pi}{4}[$ are isomorphic to $\operatorname{Sp}(1)^{3}$. Finally,

$$
\gamma\left(\frac{\pi}{4}\right)=\iota_{\mathbb{O}}\left(\begin{array}{l}
0 \\
0 \\
1
\end{array}\right)
$$

It is easy to see that the embedding $\iota_{\mathbb{O}}$ is equivariant with respect to the $\operatorname{Sp}(3) \times$ $\operatorname{Sp}(1)$-action and hence $\iota_{\mathbb{O}}$ parametrizes the orbit through $\gamma\left(\frac{\pi}{4}\right)$.

Remark 1.1. The compositions of the Hopf fibrations $\mathbb{S}^{2} \rightarrow \mathbb{R P}^{2}$ and $\mathbb{S}^{5} \rightarrow \mathbb{C P}^{2}$ with the inclusion maps $\mathbb{R P}^{2} \hookrightarrow \mathbb{C P}^{2}$ and $\mathbb{C P}^{2} \hookrightarrow \mathbb{H P}^{2}$ are null-homotopic. This follows immediately from the commutative diagram

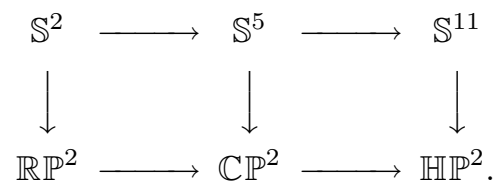

In contrast, the composition of the Hopf fibration $\mathbb{S}^{11} \rightarrow \mathbb{H} \mathbb{P}^{2}$ with the inclusion map $\mathbb{H P}^{2} \hookrightarrow \mathbb{O P}^{2}$ represents a generator or the third power of a generator of $\pi_{11}\left(\mathbb{O P}^{2}\right)$.

Remark 1.2. Suppose that $\mathbb{C P}^{2}, \mathbb{H} \mathbb{P}^{2}$, and $\mathbb{O P}^{2}$ are equipped with the standard metrics for which the sectional curvature varies between 1 and 4 . The orbit $S_{\mathbb{C}}$ inherits a metric with constant curvature 2. The orbits $S_{\mathbb{H}}$ and $S_{\mathbb{O}}$ inherit the unique metrics for which

$$
\mathbb{S}_{1 / 2}^{1} \rightarrow S_{\mathbb{H}} \rightarrow \mathbb{C P}_{1 / \sqrt{2}}^{2} \quad \text { and } \quad \mathbb{S}_{1 / 2}^{3} \rightarrow S_{\mathbb{O}} \rightarrow \mathbb{H P}_{1 / \sqrt{2}}^{2}
$$


are Riemannian fibrations. Here, the fibers are euclidean spheres with radius $\frac{1}{2}$ and the base spaces are projective planes with diameter $\frac{\pi}{2 \sqrt{2}}$. The sectional curvature of the metrics on $S_{\mathbb{H}}$ and $S_{\mathbb{O}}$ ranges from 1 to 5 and we have

$$
\operatorname{vol}\left(S_{\mathbb{C}}\right)=2 \cdot \operatorname{vol}\left(\mathbb{S}_{1 / 2}^{2}\right), \quad \operatorname{vol}\left(S_{\mathbb{H}}\right)=4 \cdot \operatorname{vol}\left(\mathbb{S}_{1 / 2}^{5}\right), \quad \operatorname{vol}\left(S_{\mathbb{O}}\right)=16 \cdot \operatorname{vol}\left(\mathbb{S}_{1 / 2}^{11}\right) .
$$

The metric on $S_{\mathbb{O}} \approx \mathbb{S}^{11}$ is the up to scaling unique metric that comes by submersion from a biinvariant metric on $\operatorname{Sp}(3)$.

Remark 1.3. The embeddings $\iota_{\mathbb{C}}, \iota_{\mathbb{H}}$, and $\iota_{\mathbb{O}}$ are harmonic with respect to any $\mathrm{SO}(3) \times \mathbb{Z}_{2}, \mathrm{SU}(3) \times \mathrm{U}(1)$, and $\mathrm{Sp}(3) \times \mathrm{Sp}(1)$-invariant metric on $\mathbb{S}^{2}, \mathbb{S}^{5}$, and $\mathbb{S}^{11}$. It is not difficult to check that the equivariance of the embeddings enforces the tension field to vanish. Note that the second suspension of the Hopf fibration $\mathbb{S}^{3} \rightarrow \mathbb{S}^{2}$ and the fourth suspension of the Hopf fibration $\mathbb{S}^{7} \rightarrow \mathbb{S}^{4}$ are harmonic maps if the suspension is done suitably [14]. Hence, the compositions of these maps with the totally geodesic inclusions $\mathbb{H P}^{1} \hookrightarrow \mathbb{H P}^{2}$ and $\mathbb{O P}^{1} \hookrightarrow \mathbb{O} \mathbb{P}^{2}$ yield other harmonic representatives of the generators of $\pi_{5}\left(\mathbb{H}^{2}\right)$ and $\pi_{11}\left(\mathbb{O P}^{2}\right)$.

Remark 1.4. The actions of $\mathrm{SO}(3) \times \mathbb{Z}_{2}$ on $\mathbb{C P}^{2}, \mathrm{U}(3)$ on $\mathbb{H}^{2}$, and $\operatorname{Sp}(3) \times_{\mathbb{Z}_{2}} \operatorname{Sp}(1)$ on $\mathbb{O P}^{2}$ have also been studied recently in [1], which deals with the diffeomorphisms $\mathbb{C P}^{2} / \mathbb{Z}_{2} \approx \mathbb{S}^{4}, \mathbb{H} \mathbb{P}^{2} / \mathrm{U}(1) \approx \mathbb{S}^{7}$, and $\mathbb{O P}^{2} / \mathrm{Sp}(1) \approx \mathbb{S}^{13}$ and their generalizations.

\section{THE CLASSIFICATION}

In this section we determine all orbits of isometric actions on the projective planes $\mathbb{C P}^{2}, \mathbb{H} \mathbb{P}^{2}$, and $\mathbb{O P}^{2}$ that are covered by spheres and represent nontrivial elements in the corresponding homotopy groups. It suffices to consider actions of connected closed subgroups of the isometry group for this problem. Moreover, we use the following fact: If two subgroups are conjugate by some element $\psi$ in the isometry group then the isometry $\psi$ maps each orbit of the first subgroup isometrically to an orbit of the second subgroup. Therefore, we can choose a fixed representative for any conjugacy class of subgroups. We will in particular do this for the maximal connected subgroups.

It is well-known that the identity components of the isometry groups of the projective planes are $\operatorname{PSU}(3), \operatorname{Sp}(3) /\{ \pm \mathbb{1}\}$, and $\mathrm{F}_{4}$, respectively. For convenience we work with $\mathrm{SU}(3)$ and $\operatorname{Sp}(3)$ instead of $\operatorname{PSU}(3)$ and $\operatorname{Sp}(3) /\{ \pm \mathbb{1}\}$. The maximal connected subgroups of $\mathrm{SU}(3), \mathrm{Sp}(3)$, and $\mathrm{F}_{4}$ with rank $\geq 2$ in the last two cases are the following (see [4], [12]):

\begin{tabular}{|c|cccc|}
\hline $\mathrm{SU}(3)$ & $\mathrm{U}(2)$ & $\mathrm{SO}(3)$ & \\
\hline $\mathrm{Sp}(3)$ & $\mathrm{Sp}(1) \times \mathrm{Sp}(2)$ & $\mathrm{U}(3)$ & $\mathrm{SO}(3) \times \mathrm{SO}(3)$ & \\
\hline $\mathrm{F}_{4}$ & $\mathrm{Spin}(9)$ & $\mathrm{Sp}(3) \times \times_{\mathbb{Z}_{2}} \mathrm{Sp}(1)$ & $\mathrm{SU}(3) \times \times_{\mathbb{Z}_{3}} \mathrm{SU}(3)$ & $\mathrm{G}_{2} \times \mathrm{SO}(3)$ \\
\hline
\end{tabular}

The first maximal subgroup in each case is the isotropy group of a point $p_{0}$ in the corresponding projective plane. The isotropy group acts transitively on the distance spheres to the point $p_{0}$ and the distance spheres finally collapse to the cut locus of $p_{0}$, i.e., to a projective line. Since inclusions of spheres of lower dimension 
into spheres of higher dimension are always null-homotopic, the only homotopically nontrivial orbit for any subgroup of the isotropy group is the cut locus of $p_{0}$ itself.

The actions of $\mathrm{SO}(3)$ on $\mathbb{C P}^{2}, \mathrm{U}(3)$ on $\mathbb{H P}^{2}$, and $\operatorname{Sp}(3) \times_{\mathbb{Z}_{2}} \operatorname{Sp}(1)$ on $\mathbb{O P}^{2}$ were explicitely given in the previous section. We first consider actions of subgroups of $\operatorname{Sp}(3) \times \operatorname{Sp}(1)$ on $\mathbb{O P}^{2}$ and any homotopically nontrivial orbit that is covered by a sphere. The dimension of this orbit is $\geq 8$. Hence, it cannot be contained in $\mathbb{H P}^{2}$. If the orbit is contained in $S_{\mathbb{O}} \approx \mathbb{S}^{11}$, it has to be identical with $S_{\mathbb{O}}$. If the orbit is neither contained in $\mathbb{H}^{2}$ nor in $S_{\mathbb{O}}$, the isotropy group of any point in the orbit is isomorphic to a subgroup of the principal isotropy group of the $\operatorname{Sp}(3) \times \operatorname{Sp}(1)$ action, i.e., to a subgroup of $\operatorname{Sp}(1)^{3}$. It follows from the classification of transitive actions on spheres (see [3], p. 179) that this can only happen if the dimension of the orbit is $\leq 7$, a contradiction. Analogous arguments apply to actions of subgroups of $\mathrm{U}(3)$ on $\mathbb{H P}^{2}$ and of subgroups of $\mathrm{SO}(3)$ on $\mathbb{C P}^{2}$ and show that $S_{\mathbb{C}}$ and $S_{\mathbb{H}}$ are the only relevant orbits of these actions.

Finally, subgroups of $\mathrm{SO}(3) \times \mathrm{SO}(3), \mathrm{SU}(3) \times{ }_{\mathbb{Z}_{3}} \mathrm{SU}(3)$, and $\mathrm{G}_{2} \times \mathrm{SO}(3)$ can only act transitively on spheres of dimension $\leq 2, \leq 5$, and $\leq 6$, respectively. Thus they do not contribute any orbit that is relevant to our problem and we have finished the classification.

Remark 2.1. The isometry groups of the projective planes are maximal compact subgroups of the projective motion groups. In the classification nothing changes if one considers actions of subgroups of the projective motion groups instead of isometric actions. The reason is a well-known theorem of Montgomery: If a connected Lie group acts transitively on a compact manifold with finite fundamental group (in our case the orbit in question) then a compact subgroup acts transitively as well.

\section{INTERSECTIONS AND HOPF FIBRATIONS}

As we have seen in Section 1 , the singular orbits $S_{\mathbb{C}}, S_{\mathbb{H}}$, and $S_{\mathbb{O}}$ are the sets of points with maximal distance to $\mathbb{R P}^{2} \subset \mathbb{C P}^{2}, \mathbb{C P}^{2} \subset \mathbb{H}^{2}$, and $\mathbb{H}^{2} \subset \mathbb{O P}^{2}$. This leads to the question whether the Hopf fibrations $\mathbb{S}^{2} \rightarrow \mathbb{R P}^{2}, \mathbb{S}^{5} \rightarrow \mathbb{C P}^{2}$, and $\mathbb{S}^{11} \rightarrow \mathbb{H} \mathbb{P}^{2}$ occur geometrically in $\mathbb{C P}^{2}, \mathbb{H}^{2}$, and $\mathbb{O P} \mathbb{P}^{2}$.

Theorem 2. The intersection of the cut locus of a point $p \in \mathbb{O P}^{2}$ with the singular orbit $\mathbb{H P}^{2}$ consists of a quaternionic projective line if $p \in \mathbb{H} \mathbb{P}^{2}$ and of a unique point $\pi(p)$ otherwise. The induced map $\pi \circ \iota_{\mathbb{O}}: \mathbb{S}^{11} \rightarrow \mathbb{H} \mathbb{P}^{2}$ is the Hopf fibration. The fiber $\pi^{-1}(q)$ is a totally geodesic $\mathbb{S}^{3}$ in $\mathbb{O P}^{2}$ given by the intersection of the cut locus of $q$ with $S_{\mathbb{O}}$. The intersection of the cut locus of an arbitrary point $p \in \mathbb{O P}^{2}$ with the singular orbit $S_{\mathbb{O}}$ is diffeomorphic to $\mathbb{S}^{3}$ if $p \notin S_{\mathbb{O}}$ and consists of just one point if $p \in S_{\mathbb{O}}$, namely, of the antipode of $p$ in $S_{\mathbb{O}}$.

Proof. It is easy to see from (4) that the cut locus of the point $p_{0} \in \mathbb{O P}^{2}$ consists precisely of the points $p \in \mathbb{O P}^{2}$ with $\left(\mathbb{1}+p_{0}\right) \circ(\mathbb{1}+p)=0$. This shows that a point $p_{2} \in \mathbb{O P}^{2}$ is in the cut locus of a point $p_{1} \in \mathbb{O P}^{2}$ if and only if $\left(\mathbb{1}+p_{1}\right) \circ\left(\mathbb{1}+p_{2}\right)=0$, since this relation is invariant under the automorphism group $\mathrm{F}_{4}$ of the Jordan algebra $\operatorname{Herm}_{\mathbb{O}}(3)$. 
Because of the $\operatorname{Sp}(3) \times \operatorname{Sp}(1)$-invariance of $\mathbb{H}^{2}$ and $S_{\mathbb{O}}$ we just have to compute the intersections of these orbits with the cut loci of points on the geodesic segment $\gamma:\left[0, \frac{\pi}{4}\right] \rightarrow \mathbb{O P}^{2}$ given in (7). We parametrize $\mathbb{H P}^{2}$ and $S_{\mathbb{O}}$ by the equivariant maps $h_{\mathbb{H}}: \mathbb{S}^{11} \rightarrow \mathbb{H} \mathbb{P}^{2}, u \mapsto\left(2 u \bar{u}^{t}-\mathbb{1}\right) \oplus 0$ and $\iota_{\mathbb{O}}: \mathbb{S}^{11} \rightarrow \mathbb{O P}^{2}, u \mapsto\left(-u \bar{u}^{t}\right) \oplus u$. A point $h_{\mathbb{H}}(u)$ in $\mathbb{H}^{2}$ is contained in the cut locus of $\gamma(t)$ if and only if

$$
(\mathbb{1}+\gamma(t)) \circ\left(u \bar{u}^{t} \oplus 0\right)=0 .
$$

Straigthforward computations show that for $t=0$ this condition is equivalent to $u_{1}=0$, which defines a quaternionic projective line. For $0<t \leq \frac{\pi}{4}$ the condition is equivalent to $u_{1}=u_{2}=0,\left|u_{3}\right|=1$, which defines a single point. It follows from (8) and the equivariance of $\iota_{\mathbb{O}}$ that $\pi \circ \iota_{\mathbb{O}}$ is the Hopf fibration.

A point in the orbit $S_{\mathbb{O}}$ is contained in the cut locus of $\gamma(t)$ if and only if

$$
(\mathbb{1}+\gamma(t)) \circ\left(\left(\mathbb{1}-u \bar{u}^{t}\right) \oplus u\right)=0 .
$$

For $t \in\left[0, \frac{\pi}{4}\right]$ this condition is equivalent to

$$
\left|u_{1}\right|^{2}=1-\tan ^{2} t, \quad u_{2}=0, \quad \text { and } \quad u_{3}=-\tan t .
$$

For $t=0$ this becomes $\left|u_{1}\right|^{2}=1, u_{2}=u_{3}=0$, which is the Hopf fiber $h_{\mathbb{H}}^{-1}(\gamma(0))$. Using the isometric identification of the cut locus of $p_{0}$ with $\mathbb{S}_{1 / 2}^{8}$ given in (4) we see that the intersection of $S_{\mathbb{O}}$ with the cut locus of $p_{0}=\gamma(0)$ is a totally geodesic $\mathbb{S}_{1 / 2}^{3}$.

Remark 3.1. To projective geometers the orbits $S_{\mathbb{C}}, S_{\mathbb{H}}$, and $S_{\mathbb{O}}$ are known as the sets of absolute points of the planar polarities [13]. It is also known that the intersections of $S_{\mathbb{C}}, S_{\mathbb{H}}$, and $S_{\mathbb{O}}$ with generic lines are diffeomorphic to $\mathbb{S}^{0}, \mathbb{S}^{1}$, and $\mathbb{S}^{3}$, respectively. In $\mathbb{C P}^{2}$ this fact is a special consequence of Bezout's theorem and it can easily be used to prove that the quadric represents twice a generator of $\pi_{2}\left(\mathbb{C P}^{2}\right)$. At this point a natural question arises: Is it possible to prove that $S_{\mathbb{H}}$ and $S_{\mathbb{O}}$ generate $\pi_{5}\left(\mathbb{H}^{2}\right)$ and $\pi_{11}\left(\mathbb{O P}^{2}\right)$ from the intersection behaviour of these orbits with projective lines? In combination with our deformation such an argument could provide a unified geometric proof for the fact that the suspensions of the Hopf fibrations $\mathbb{S}^{3} \rightarrow \mathbb{S}^{2}$ and $\mathbb{S}^{7} \rightarrow \mathbb{S}^{4}$ generate the stable homotopy groups $\pi_{n+1}\left(\mathbb{S}^{n}\right)$ and $\pi_{n+3}\left(\mathbb{S}^{n}\right)$.

\section{ACKNOWLEDGEMENTS}

The authors would like to thank Uwe Abresch, Andreas Gastel, Linus Kramer, and Hong Van Le for comments on the manuscript.

\section{REFERENCES}

[1] M. Atiyah, J. Berndt, Projective planes, Severi varities, and spheres, J. Differential Geom., to appear.

[2] J. C. Baez, The octonions, Bull. Am. Math. Soc. 39 (2002), 145-205.

[3] A.L. Besse, Einstein manifolds, Ergebnisse der Mathematik und ihrer Grenzgebiete 10, Springer, Berlin, 1987. 
[4] E. B. Dynkin, Maximal subgroups of the classical groups, Am. Math. Soc. Transl., II. Ser. 6 (1957), 245-378.

[5] H. Freudenthal, Oktaven, Ausnahmegruppen und Oktavengeometrie, Geom. Dedicata 19 (1985), 7-63.

[6] F. R. Harvey, Spinors and Calibrations, Perspectives in Mathematics 9, Academic Press, Boston, 1990.

[7] W. Y. Hsiang, H. B. Lawson, Minimal submanifolds of low cohomogeneity, J. Differ. Geom. 5 (1971), 1-38.

[8] S.T. Hu, Homotopy theory, Pure and Applied Mathematics VIII, Academic Press, New York, 1959.

[9] K. Iwata, Compact transformation groups on rational cohomology Cayley projective planes, Tohoku Math. J. 33 (1981), 429-442.

[10] P. Jordan, Über eine nicht-desarguessche ebene projektive Geometrie, Abh. Math. Sem. Univ. Hamburg 16 (1949), 74-76.

[11] Y. Ohnita, Stable minimal submanifolds in compact rank one symmetric spaces, Tohoku Math. J. 38 (1986), 199-217.

[12] A. L. Onishchik, E. B. Vinberg, V. V. Gorbatsevich, Lie groups and Lie algebras III, Encyclopaedia of Mathematical Sciences 41, Springer, Berlin, 1994.

[13] H. Salzmann, D. Betten, T. Grundhöfer, H. Hähl, R. Löwen, M. Stroppel, Compact projective planes, Expositions in Mathematics, 21, Walter de Gruyter, Berlin, 1995.

[14] R. T. Smith, Harmonic mappings of spheres, Amer. J. Math. 97 (1975), 364-385.

[15] G. W. Whitehead, Elements of Homotopy Theory, Graduate Texts in Mathematics 61, Springer-Verlag, Berlin, 1978.

Ruhr-Universität Bochum, Fakultät für Mathematik, D-44780 Bochum, Germany

E-mail address: puttmann@math.ruhr-uni-bochum.de

IMECC, UNICAMP, C.P. 6065, 13083-970 CAMPINAS, SP, BRAZIL

E-mail address: rigas@ime.unicamp.br 